\title{
Pharmacokinetics of Orally Administered Acetaminophen in Man
}

\author{
Kenneth S. Albert, ${ }^{1}$ Allen J. Sedman, ${ }^{1}$ and John G. Wagner ${ }^{1,2}$
}

Received Jan. 17, 1974-Final July 22, 1974

\begin{abstract}
Average and individual sets of plasma concentration-time data for acetaminophen following two oral treatments were simultaneously fitted to the integrated equation describing the two-compartment open model with first-order absorption and lag time. The nonlinear least-squares program NONLIN and an IBM $360 / 67$ digital computer were employed to estimate nine parameters $\left(\mathrm{k}_{\mathrm{A}}, \mathrm{k}_{\mathrm{B}}, \mathrm{C}_{\mathrm{A}}^{0}, \mathrm{C}_{\mathrm{B}}^{0}\right.$, $\mathrm{k}_{12}, \mathrm{k}_{21}, \mathrm{k}_{\mathrm{el}}, \mathrm{t}_{\mathrm{O}_{\mathrm{A}}}$ and $\mathrm{t}_{\mathrm{0}_{\mathrm{B}}}$ ). When the mean plasma concentrations were weighted according to the inverse of their variances, the parameter estimates more accurately reflected those for individual subjects in the disposition portion of the model. Depending on the relative magnitudes of the disposition rate constants $\left(\mathrm{k}_{12}, \mathrm{k}_{21}\right.$, and $\left.\mathrm{k}_{\mathrm{e}}\right)$, the one-compartment open model can be used to predict equilibrium-state plasma levels even though the drug is really "two compartment." Equations are presented which show when the one-compartment approximation is justified. Equations are also presented for calculation of loading doses for multiple dose regimens of any drug obeying the twocompartment open model and the equations are applied to acetaminophen.
\end{abstract}

KEY WORDS: acetaminophen; oral administration; plasma levels; simultaneous fitting; weighting factor; central compartment correction factor.

\section{INTRODUCTION}

The utility of pharmacokinetics for quantification of drug absorption and disposition is well documented (1). The approach permits predictions regarding drug dosage adjustment and, in addition, allows for comparison of the effects of dosage form variables on therapeutic efficacy and/or bioavailability. In a recent study, Albert et al. (2) noted differences in rates of

These studies were supported by a contract from the R. P. Scherer Corporation and partly by Public Health Service Grant 5-P11-GM15559.

${ }^{1}$ College of Pharmacy and Upjohn Center for Clinical Pharmacology, The University of Michigan, Ann Arbor, Michigan 48104.

${ }^{2}$ Address reprint requests to Dr. John G. Wagner, Upjohn Center for Clinical Pharmacology, The University of Michigan, Ann Arbor, Michigan 48104. 
absorption of acetaminophen when the drug was administered separately to ten healthy volunteers both as a commercial tablet and as a specially formulated soft gelatin capsule. In this report, the pharmacokinetic model which was elaborated from the plasma concentration-time data will be discussed.

\section{EXPERIMENTAL}

\section{Treatment of Data}

The study conditions were described in a previous report (2). Average and individual sets of plasma concentration-time data obtained following two oral treatments of acetaminophen were simultaneously fitted to the integrated equation describing the two-compartment open model with first-order absorption and lag time $t_{0}$ (Model I and equations A1 and A2 of the Appendix). The nonlinear least-squares program NONLIN and an IBM 360/67 digital computer were employed. The nine parameters estimated, corresponding to Model $\mathrm{I}$, were $k_{A}, k_{B}, k_{12}, k_{21}, k_{\mathrm{el}}, C_{A}^{0}\left(F_{A} D / V_{1}\right)$, $C_{B}^{0}\left(F_{B} D / V_{1}\right), t_{0_{A}}$, and $t_{0_{B}}$ where the subscripts $A$ and $B$ refer to treatments A (soft gelatin capsule) and $\mathbf{B}$ (commercially available tablet).

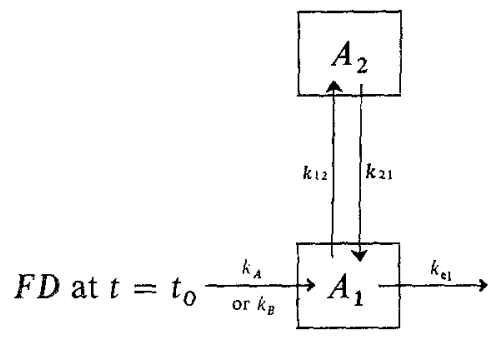

Model I

Estimates of the parameters were obtained by minimizing the sums of the weighted deviations, i.e., $\sum_{i=1}^{n} w_{i}\left(\hat{C}_{i}-C_{i}\right)^{2}$, where $w_{i}$ is the weighting factor, $\hat{C}_{i}$ is the estimated concentration, and $C_{i}$ is the observed concentration at time $t_{i}$. The mean plasma concentrations in separate trials were weighted (a) equally; (b) according to the inverse of their concentrations, $1 / \bar{C}_{i} ;$ (c) according to the inverse of the square of their concentrations, $1 /\left(\bar{C}_{i}\right)^{2}$; and (d) according to the inverse of their individual variances, $1 / \sigma_{i}^{2}$. Individual subject concentrations were weighted according to $1 / C_{i}$ and $1 / C_{i}^{2}$.

Criteria used to assess adequate fit of the data to the model were (a) $r^{2}$, the coefficient of determination, equivalent to $\left(\Sigma \mathrm{Obs}^{2}-\Sigma \mathrm{Dev}^{2}\right) / \Sigma \mathrm{Obs}^{2}$; 
(b) Corr, the correlation coefficient for the linear regression of the modelpredicted plasma concentration vs. observed plasma concentration; (c) lack of trends or regions of poor fit as delineated by a plot of the weighted residual (weight $\times \%$ deviation) vs. observed plasma concentration; and (d) the magnitudes of the coefficients of variation of the estimated parameters (standard deviation of the estimate/estimated parameter).

\section{Weighting Considerations}

Equation 1, which describes a relationship between variance and concentration, may be used to test a feasible weighting factor $\left(w_{i}\right)$ for fitting individual subject plasma concentrations to a model based on the mean plasma levels of the panel of subjects to which the individuals belong:

$$
\sigma^{2}=a \cdot(\bar{C})^{n}
$$

where $\sigma^{2}$ is the variance corresponding to mean concentration $\bar{C}$ and $a$ and $n$ are constants. Taking logarithms of both sides of equation 1 gives equation 2 :

$$
\ln \sigma^{2}=\ln a+n \ln \bar{C}
$$

Plotting $\ln \sigma^{2}$ vs. $\ln \bar{C}$ generates a straight line with intercept $\ln a$ (corresponding to $\bar{C}=1$ ) and slope $n$. If $n=1, w_{i}=1 / C_{i}$; if $n=2, w_{i}=1 / C_{i}^{2}$. This is a reasonable method for estimating the appropriate weights for an individual subject set of plasma level data since there is only one observation at each time. Adherence to equation 2 indicates that the trends in variance across a concentration-time curve are due only to the magnitude of the plasma concentrations and are independent of time or other effects. If the correlation coefficient is very high for the averages (i.e., for the mean plasma levels) when equation 2 is applied, then one could use $w_{i}=1 / a(\bar{C})^{n}$ to fit average plasma concentrations, or one could still use the weighting factor $1 / \sigma_{i}^{2}$ (as we chose to do) where $\sigma_{i}^{2}$ is calculated from the panel's concentrations at each time $t_{i}$. In using the variance of mean plasma concentrations, the sampling times have to be the same for each subject, as they were in our study.

\section{Theoretical Treatment of Disposition Rate Parameters}

The microscopic rate constants $k_{12}, k_{21}$, and $k_{\mathrm{e} 1}$ which describe the disposition portion of Model I can be used to estimate how well $C, t$ data can be approximated by the one-compartment open model. Equation 3 , applicable to the two-compartment open model with rapid intravenous injection $^{3}$ (see Appendix), shows that as $B /(A+B) \rightarrow 1$, the model reduces

\footnotetext{
${ }^{3}$ The same conclusions would apply for first-order absorption.
} 
to the one-compartment open model, because the first term on the right-hand side vanishes since $A \rightarrow 0$.

$$
C_{1} / C_{1}^{0}=[A /(A+B)] e^{-\alpha t}+[B /(A+B)] e^{-\beta t}
$$

In equation $3, B /(A+B)=\left(k_{21}-\beta\right) /(\alpha-\beta)$ and $C_{1}^{0}=A+B$. The "collapsing," if it occurs, depends on the relative magnitudes of $k_{21}, \alpha$, and $\beta$. However, specifically, as $k_{21} \rightarrow \alpha$, "collapsing" occurs. Equation 3 also indicates that if $k_{21} \gg \beta$ and $\alpha \gg \beta$, then the ratio $B /(A+B) \simeq \beta / k_{\text {e1 }}=$ $k_{21} / \alpha$.

Sometimes, for drugs which actually obey the two-compartment open model, the one-compartment open model can be used to predict average steady-state amounts of drug in the body during multiple dosing. For the one-compartment model,

$$
\vec{A}_{\infty}=F D / \beta \tau
$$

where $\bar{A}_{\infty}$ represents the average amount of drug in the body at the equilibrium state and $\tau$ represents the dosage interval. An analogous expression, derived in the Appendix, can be written for the two-compartment open model :

$$
\bar{A}_{\infty}=\left[\beta / k_{\mathrm{el}}\left(1+k_{12} / k_{21}\right) F D\right] / \beta \tau
$$

It is apparent that when the factor $\beta / k_{\mathrm{c} 1}\left(1+k_{12} / k_{21}\right) \rightarrow 1$, equation 5 reduces to equation 4 and the one-compartment open model would accurately predict $\bar{A}_{\infty}$. This factor may be viewed as a central compartment correction factor (CCCF) for the two-compartment open model compared with the one-compartment open model.

Equation 5 can be rearranged to equation 6 to provide the "accumulation ratio" $\left(R_{A}\right)$ for the two-compartment open model as described by Wagner (3):

$$
R_{A}=\bar{A}_{\infty} / F D=\left(1+k_{12} / k_{21}\right) / k_{\mathrm{el}} \tau
$$

$R_{A}$ is equal to the average amount of drug in the body during a dosage interval at the equilibrium state divided by the amount of drug absorbed following a single maintenance dose. Since $R_{A}$ can be estimated from the parameters of the two-compartment model, the loading dose for a multiple dosing regimen can be calculated using equation 7 :

$$
\text { Loading dose }=R_{A} \cdot \text { maintenance dose }
$$

The central compartment correction factor is of particular importance in estimating loading doses since the loading dose using the two-compartment equation (equation 6) will always be less than the loading dose estimated using the one-compartment equation (equation 4) for equal main- 
tenance doses. It is possible that with some drugs toxicity problems are caused by use of the inappropriate model to estimate the loading dose.

\section{RESULTS}

Table I lists the pharmacokinetic parameters obtained by simultaneously fitting mean plasma concentrations of acetaminophen to Model I and equations A1 and A2 of the Appendix. In such simultaneous fitting, the weighted sum of squared deviations of both sets of data is minimized. With the exception of $w_{i}=1 / \sigma_{i}^{2}$, the weighting factors had little influence on the parameter estimates and their corresponding coefficients of variation. Use of $w_{i}=1 / \sigma_{i}^{2}$ gave different values for the parameters and higher coefficients of variation of the estimates.

Table II gives the parameter estimates from simultaneous fitting of the two sets of $C, t$ data for each subject to Model I employing the weighting factors $1 / C_{i}$ and $1 / C_{i}^{2}$. Equal weighting was not used since the error in the

Table I. Parameter Estimates and Coefficients of Variation for Mean Plasma Concentrations of Acetaminophen

\begin{tabular}{lcccc}
\hline & \multicolumn{3}{c}{ Estimated parameters and coefficients of variation } \\
\cline { 2 - 5 } Parameter & $\begin{array}{c}\text { Equal } \\
\text { weighting }\end{array}$ & $\begin{array}{c}1 / \bar{C}_{i} \\
\text { weighting }\end{array}$ & $\begin{array}{c}\left.1 / \bar{C}_{i}\right)^{2} \\
\text { weighting }\end{array}$ & $\begin{array}{c}1 / \sigma_{i}^{2} \\
\text { weighting }\end{array}$ \\
\hline$k_{A}\left(\mathrm{hr}^{-1}\right)$ & 0.991 & 1.04 & 1.07 & 0.764 \\
& $(0.157)$ & $(0.138)$ & $(0.138)$ & $(0.268)$ \\
$k_{B}\left(\mathrm{hr}^{-1}\right)$ & 2.03 & 2.22 & 2.45 & 1.27 \\
& $(0.211)$ & $(0.215)$ & $(0.268)$ & $(0.372)$ \\
$k_{12}\left(\mathrm{hr}^{-1}\right)$ & 0.106 & 0.0827 & 0.0707 & 0.319 \\
& $(0.570)$ & $(0.494)$ & $(0.406)$ & $(0.696)$ \\
$k_{21}\left(\mathrm{hr}^{-1}\right)$ & 0.203 & 0.262 & 0.246 & 0.499 \\
& $(0.586)$ & $(0.382)$ & $(0.365)$ & $(0.206)$ \\
$k_{\mathrm{e} 1}\left(\mathrm{hr} r^{-1}\right)$ & 0.324 & 0.321 & 0.310 & 0.472 \\
$C_{A}^{0}(\mu \mathrm{g} / \mathrm{ml})$ & $(0.146)$ & $(0.103)$ & $(0.097)$ & $(0.339)$ \\
& 9.98 & 9.51 & 9.18 & 13.7 \\
$C_{B}^{0}(\mu \mathrm{g} / \mathrm{ml})$ & $(0.124)$ & $(0.107)$ & $(0.109)$ & $(0.337)$ \\
$t_{0 A}(\mathrm{hr})$ & 9.38 & 8.96 & 8.56 & 12.9 \\
& $(0.112)$ & $(0.110)$ & $(0.110)$ & $(0.336)$ \\
$t_{0 B}(\mathrm{hr})$ & 0.164 & 0.165 & 0.165 & 0.165 \\
$r^{2}$ & $(0.108)$ & $(0.011)$ & $(0.017)$ & $(0.008)$ \\
$C_{0}$ & 0.0342 & 0.0425 & 0.0498 & 0.00252 \\
$\alpha$ & $(0.705)$ & $(0.522)$ & $(0.524)$ & $(1.18)$ \\
$\beta$ & 0.998 & 0.998 & 0.998 & 0.997 \\
& 0.997 & 0.997 & 0.996 & 0.996 \\
& 0.502 & 0.496 & 0.461 & 1.07 \\
& 0.132 & 0.169 & 0.165 & 0.220 \\
\hline
\end{tabular}

${ }^{a}$ Numbers in parentheses are coefficients of variation (standard deviation/estimate) of the estimates. 


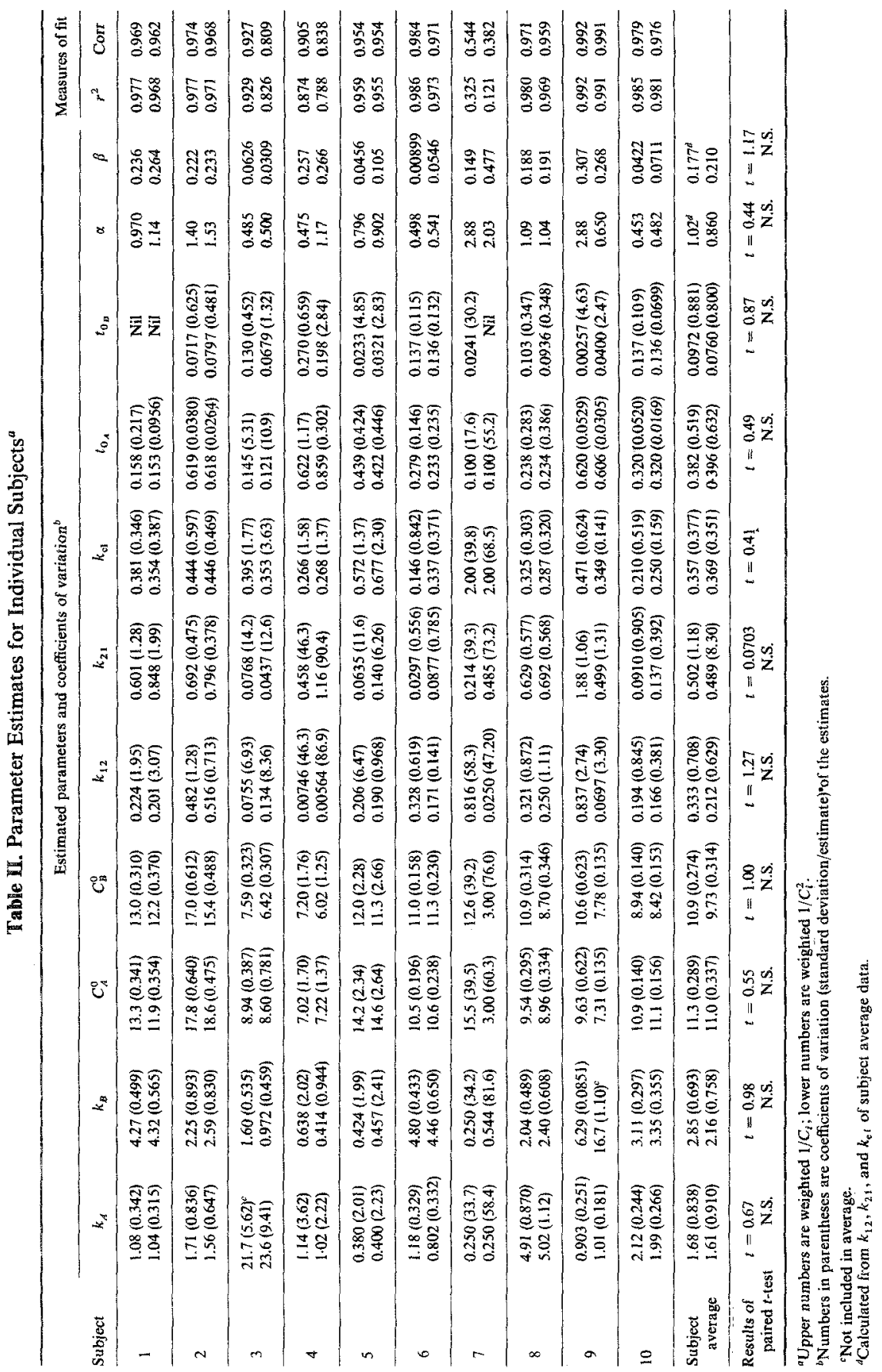




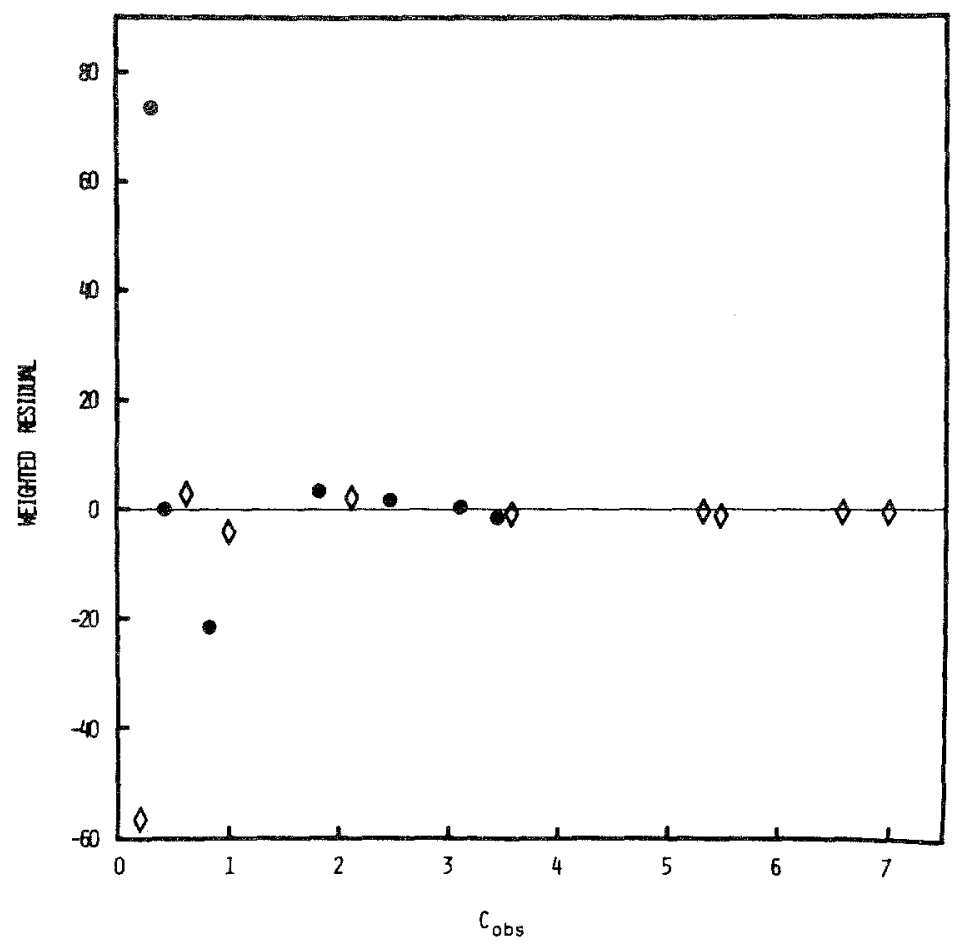

Fig. 1. Weighted residual plot for subject 9. Treatment A; $\diamond$, treatment $B$.

GLC assay was concentration dependent and this error is a part of the total error.

A paired $t$-test indicated in each case that the means of the estimates obtained using the different weighting schemes did not differ significantly. The reasonably high $r^{2}$ and Corr values indicated the applicability to the data of the two-compartment open model with first-order absorption and lag time. In addition, weighted residual plots, a typical example of which is shown in Fig. 1, gave no evidence of trends or regions of poor fit. Figures 2 and 3 graphically compare the observed and model-predicted concentrations for subjects 4 and 9 . These data represent the worst and best fits, respectively. (Although Table II shows that subject 7 gave the worst fit, the observed plasma levels indicated that it was impossible to fit these data to any linear model. Hence this subject's parameters were not included in any averages.)

A comparison of the disposition rate parameters in Tables I and II reveals that use of $w_{i}=1 / \sigma_{i}^{2}$ for the mean plasma data more accurately 
Table III. Comparison of Disposition Rate Parameters, Estimated from Mean Plasma Concentration Data, with Averages of Individual Subject Parameter Estimates

Parameter estimates

From mean plasma level curve

\begin{tabular}{lccc}
\cline { 2 - 3 } Parameter & $\begin{array}{c}\text { Average of } 1 / \bar{C}_{i} \\
\text { and } 1 /\left(\bar{C}_{i}\right)^{2} \text { data }^{a}\end{array}$ & $1 / \sigma_{i}^{2}$ & $\begin{array}{c}\text { Average of parameter from } \\
\text { individual subject fittings }\end{array}$ \\
\hline$k_{12}\left(\mathrm{hr}^{-1}\right)$ & 0.0767 & 0.319 & 0.273 \\
$k_{21}\left(\mathrm{hr}^{-1}\right)$ & 0.254 & 0.499 & 0.496 \\
$k_{\mathrm{el}}\left(\mathrm{hr}^{-1}\right)$ & 0.316 & 0.472 & 0.363 \\
$\alpha\left(\mathrm{hr}^{-1}\right)$ & 0.479 & 1.07 & 0.940 \\
$\beta\left(\mathrm{hr}^{-1}\right)$ & 0.167 & 0.220 & 0.194 \\
\hline
\end{tabular}

${ }^{a}$ Obtained by averaging columns headed " $1 / \bar{C}_{i}$ weighting" and " $1 /\left(\bar{C}_{i}\right)^{2}$ weighting" in Table I.

${ }^{b}$ Obtained by averaging the row titled "Subject average" in Table II.

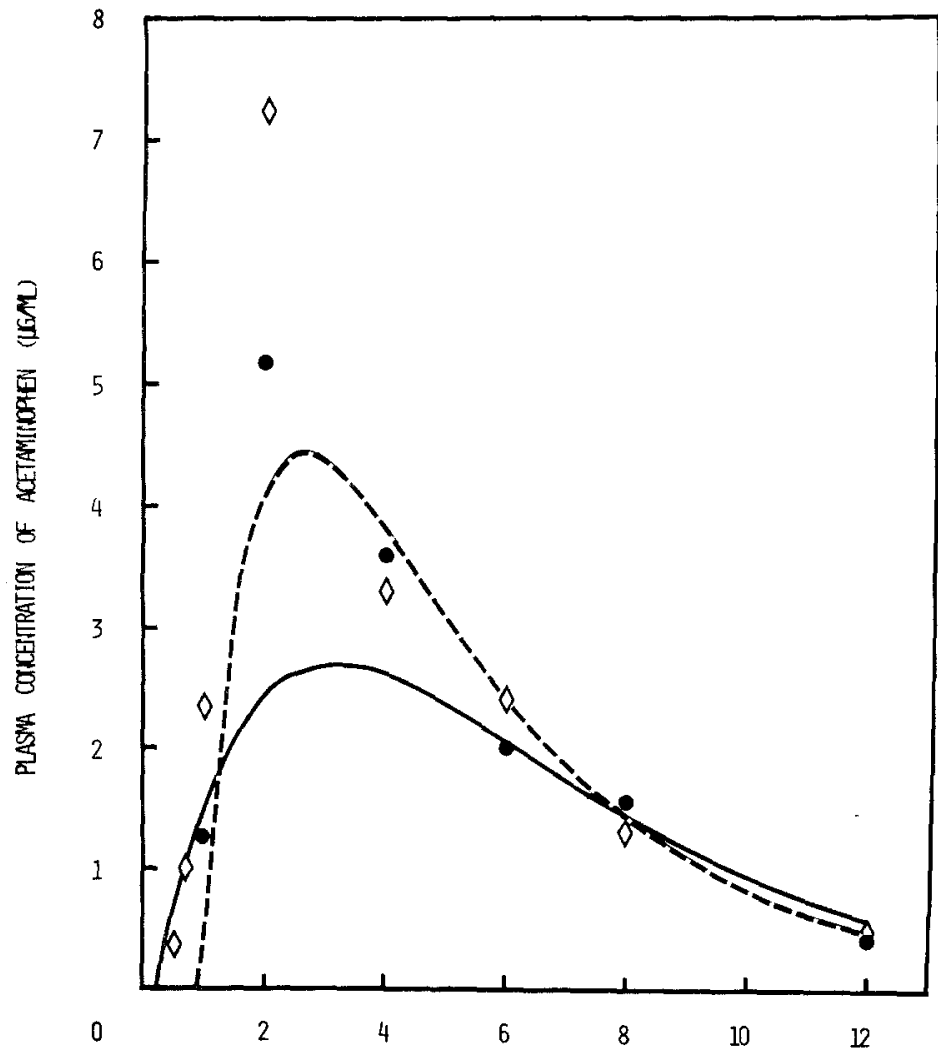

Fig. 2. Observed $v s$. model-predicted plasma concentrations of acetaminophen for subject 4 , representing the worst fit of all subjects; $w_{i}=1 / C_{i}^{2}$.

Treatment A; $\diamond-\diamond$, treatment B. 


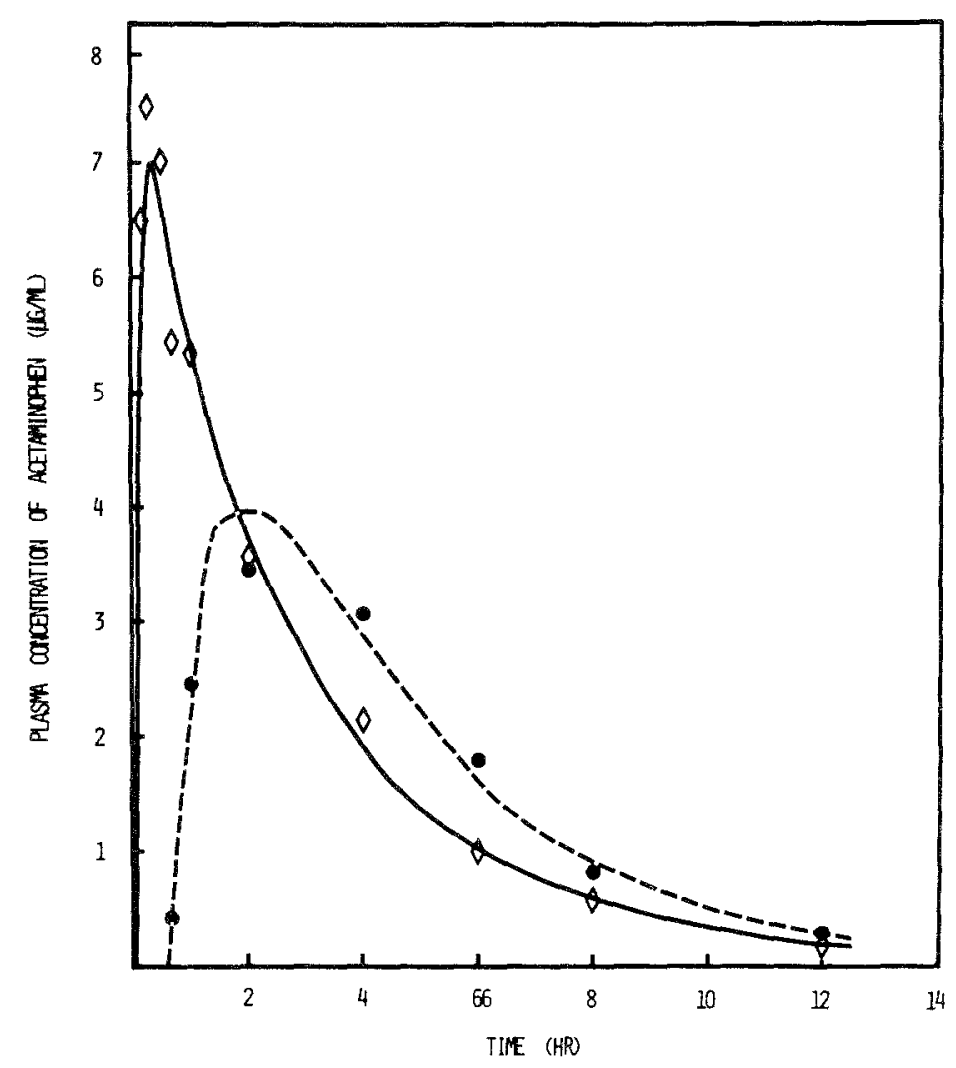

Fig. 3. Observed $v s$. model-predicted plasma concentrations of acetaminophen for subject 9 , representing the best fit of all subjects; $w_{i}=1 / C_{i}^{2}$.

Treatment A; $\diamond-\diamond$, treatment B.

reflects those derived from individual subject data. This is clearly shown in Table III. The observation is not surprising since the pattern of variances that occurs across an average blood level curve would be the same pattern expected if the same subject were administered the same treatment a large number of times. This suggests that equation 2 can be used to estimate the appropriate weight for individual subject sets of acetaminophen data using the mean plasma levels of the panel. Application of equation 2 gave $\sigma^{2}=$ $0.252(\bar{C})^{2.02}(r=0.818)$. Therefore, weighting individual subject sets of $C, t$ data according to $1 / C_{i}^{2}$ is statistically justified in this case.

Table IV lists values of $\beta / k_{\mathrm{el}}, B /(A+B)$, and $\beta / k_{\mathrm{el}}\left(1+k_{12} / k_{21}\right)$ calculated for each subject using $w_{i}=1 / C_{i}^{2}$. Since the ratio $B /(A+B)$ is generally much less than unity, acetaminophen is definitely "two compartment" and poorly approximated by the one-compartment open model. Moreover, 
Table IV. Pharmacokinetic Parameters Calculated from Disposition Rate Constants Weighting According to $1 / \bar{C}_{i}^{2}$

\begin{tabular}{|c|c|c|c|}
\hline Subject & $\beta / k_{\mathrm{el}}$ & $\beta / k_{\mathrm{e} 1}\left(1+k_{12} / k_{21}\right)$ & $B /(A+B)^{a}$ \\
\hline 1 & 0.746 & 0.923 & 0.667 \\
\hline 2 & 0.522 & 0.861 & 0.435 \\
\hline 3 & 0.0875 & 0.356 & 0.0272 \\
\hline 4 & 0.993 & 0.998 & 0.989 \\
\hline 5 & 0.155 & 0.365 & 0.0439 \\
\hline 6 & 0.162 & 0.478 & 0.0680 \\
\hline 7 & 0.238 & 0.250 & 0.0051 \\
\hline 8 & 0.666 & 0.907 & 0.590 \\
\hline 9 & 0.768 & 0.875 & 0.604 \\
\hline 10 & 0.204 & 0.451 & 0.160 \\
\hline Average $^{b}$ & 0.478 & 0.690 & 0.398 \\
\hline C.V. $(\%)$ & 69.8 & 39.0 & 85.6 \\
\hline \multirow{3}{*}{$\begin{array}{l}\text { From average plasma level curve } \\
\left(1 / \bar{C}_{i}\right)^{2} \\
1 / \sigma_{i}^{2}\end{array}$} & & & \\
\hline & 0.532 & 0.685 & 0.274 \\
\hline & 0.466 & 0.764 & 0.328 \\
\hline
\end{tabular}

${ }^{a} B /(A+B)=\left(k_{21}-\beta\right) /(\alpha-\beta)$.

${ }^{b}$ Excludes subject 7 .

since $\beta$ is not clearly small enough compared to $k_{21}$ (see Table II), $B /(A+B$ ) underestimates $\beta / k_{\mathrm{el}}$. Table IV also shows that the values of CCCF for subjects $3,5,6,7$, and 10 as well as for subject averages are much less than unity. Hence $\bar{A}_{\infty}$ of acetaminophen cannot be accurately predicted by the onecompartment open model. A large error would also occur in the estimated loading dose if the one-compartment approximation were applied to acetaminophen, because $R_{A}$ for the one-compartment model would be greater than that for the two-compartment case. For subjects 1,2, 4, 8, and 9, on the other hand, $\bar{A}_{\infty}$ can be approximated well by the one-compartment model despite the fact that the data really obey the two-compartment model. Hence the one-compartment approximation would predict, by equation 7 , that when $\tau=t_{1 / 2}$ the loading dose would be 1.44 times the maintenance dose, where $t_{1 / 2}=0.693 / \beta$.

\section{DISCUSSION}

The simultaneous fitting of plasma concentration-time data for each subject to Model I and equations A1 and A2 of the Appendix revealed that in general $k_{B}>k_{A}$. Absorption-time plots based on the Loo-Riegelman method (4) gave results consistent with first-order input kinetics. This supports and quantifies previous observations by Albert et al. (2), who reported that when acetaminophen was administered in the form of a commercial tablet it was more rapidly absorbed than when it was administered as a soft gelatin capsule. Evaluation of the data in Table II also showed that the 
apparent plasma clearance area remained constant intrasubject. Evidence for this is provided by a paired $t$-test in which a comparison of the means of the dose/area ratios following treatments $\mathrm{A}$ and $\mathrm{B}$ gave differences that tested not significant $(t=1.82, \mathrm{df}=8)$. This suggests that efficiencies of absorption of acetaminophen can be estimated by a direct comparison of areas under the $C, t$ curves rather than by invoking the corrected area procedure described by Wagner (5).

Simultaneous fitting of two or more sets of plasma data to a model is generally preferred to individual fitting since there is an appreciable increase in the degrees of freedom. The standard deviations of the estimated parameters are consequently reduced (6). Nine parameters were estimated by simultaneous fitting of between 15 and 20 data points (depending on the magnitude of $t_{0}$ ) rather than by use of six to ten data points for six parameters, as would be the case in individual fitting of these data. The parameters $k_{12}$, $k_{21}$, and $k_{\mathrm{e} 1}$ were assumed constant intrasubject from treatment to treatment. Therefore, only one value of each was estimated. Initial estimates of these disposition rate constants obtained by use of an electronic calculator indicated that, indeed, this constancy assumption was justified with acetaminophen.

Model I shows loss of drug from the inner compartment only. The data could have been fitted equally well to Model II with loss from the outer compartment or to Model III with loss from both compartments:

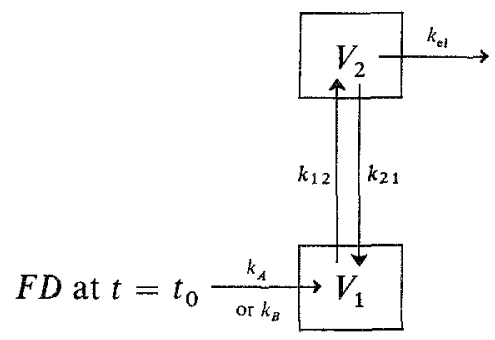

Model II

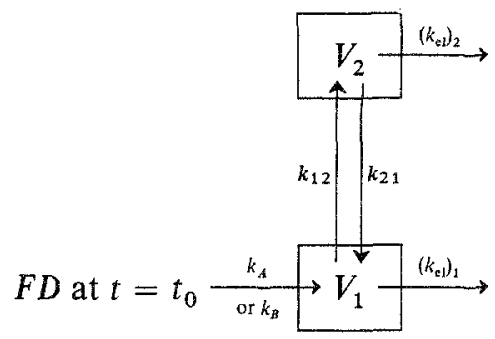


Since these models are pharmacokinetically indistinguishable when only the central compartment is available for sampling, only three rate parameters can be determined in the disposition portion of the model (7) even though four microscopic rate constants are pictorially depicted in Model III. If one wishes to calculate the values of $k_{12}, k_{21}$, and $k_{\mathrm{el}}$ for Model II or $k_{12}$, $E_{2}=k_{21}+\left(k_{\mathrm{e}}\right)_{2}$, and $\left(k_{\mathrm{el}}\right)_{1}$ for Model III from the parameters estimated for Model I, the conversion equations are readily obtained.

\section{APPENDIX}

\section{Equations Employed in Simultaneous Fitting}

For treatment A :

$C_{A}(t)=k_{A} C_{A}^{0}\left[\frac{\left(k_{21}-\alpha\right) e^{-\alpha t_{A}^{\prime}}}{\left(k_{A}-\alpha\right)(\beta-\alpha)}+\frac{\left(k_{21}-\beta\right) e^{-\beta t_{A}^{\prime}}}{\left(k_{A}-\beta\right)(\alpha-\beta)}+\frac{\left(k_{21}-k_{A}\right) e^{-k_{A} t_{A}^{\prime}}}{\left(\alpha-k_{A}\right)\left(\beta-k_{A}\right)}\right]$

where $t_{A}^{\prime}=t-t_{0_{A}}, \alpha>\beta$, and $C_{A}^{0}=$ absorbed dose $/ V_{1}$ following treatment A.

For treatment B:

$$
C_{B}(t)=k_{B} C_{B}^{0}\left[\frac{\left(k_{21}-\alpha\right) e^{-\alpha t_{B}^{\prime}}}{\left(k_{B}-\alpha\right)(\beta-\alpha)}+\frac{\left(k_{21}-\beta\right) e^{-\beta t_{B}^{\prime}}}{\left(k_{B}-\beta\right)(\alpha-\beta)}+\frac{\left(k_{21}-k_{B}\right) e^{-k_{B} t_{B}^{\prime}}}{\left(\alpha-k_{B}\right)\left(\beta-k_{B}\right)}\right]
$$

where $t_{B}^{\prime}=t-t_{0_{B}}, \alpha>\beta$, and $C_{B}^{0}=$ absorbed dose $/ V_{1}$ following treatment $B$.

In equations $\mathrm{A} 1$ and $\mathrm{A} 2$,

$$
\alpha, \beta=\frac{1}{2}\left\{\left(k_{12}+k_{21}+k_{\mathrm{el}}\right) \pm\left[\left(k_{12}+k_{21}+k_{\mathrm{el}}\right)^{2}-4 k_{21} k_{\mathrm{el}}\right]^{1 / 2}\right\}
$$

\section{Equations Employed in One-Compartment Approximations}

When elimination occurs only from the central compartment,

$$
C_{1}(t)=\frac{D}{V_{1}(\alpha-\beta)}\left[\left(k_{21}-\beta\right) e^{-\beta t}-\left(k_{21}-\alpha\right) e^{-\alpha t}\right]
$$

Equation $\mathrm{A} 4$ can be written as equation $\mathrm{A} 5$ :

$$
C_{1}(t)=A e^{-\alpha t}+B e^{-\beta t}
$$

Since

$$
D / V_{1}=A+B=C_{1}^{0}
$$

it follows that

$$
C_{1} / C_{1}^{0}=[A /(A+B)] e^{-\alpha t}+[B /(A+B)] e^{-\beta t}
$$


Therefore,

$$
B /(A+B)=\left(k_{21}-\beta\right) /(\alpha-\beta)
$$

Perrier and Gibaldi (8) showed that

$$
\bar{A}_{\infty}=\bar{C}_{\infty} \cdot V_{d_{\mathrm{ss}}}=V_{d_{\mathrm{ss}}} / V_{d_{\mathrm{arca}}} \cdot F D / \beta \tau
$$

Also,

$$
\begin{aligned}
V_{d_{\mathrm{ss}}} & =\left(1+k_{12} / k_{21}\right) V_{1} \\
V_{d_{\text {area }}} & =V_{1}\left(k_{\mathrm{el}} / \beta\right)
\end{aligned}
$$

Hence,

$$
V_{d_{\mathrm{ss}}} / V_{d_{\mathrm{area}}}=\left(\beta / k_{\mathrm{el}}\right)\left(1+k_{12} / k_{21}\right)
$$

Substituting equation $\mathrm{A} 12$ into equation $\mathrm{A} 9$ gives equation A13:

$$
\bar{A}_{\infty}=\left[\left(1+k_{12} / k_{21}\right) F D\right] / k_{\mathrm{el}} \tau
$$

\section{ACKNOWLEDGMENT}

The authors are indebted to Carl Metzler, Upjohn Company, Kalamazoo, Michigan, for the use of the program NONLIN.

\section{REFERENCES}

1. J. G. Wagner. Biopharmaceutics and Relevant Pharmacokinetics, Drug Intelligence Publications, Hamilton, Ill., 1971.

2. K. S. Albert, A. J. Sedman, P. Wilkinson, R. G. Stoll, W. J. Murray, and J. C. Wagner. Bioavailability studies of acetaminophen and nitrofurantoin. J. Clin. Pharmacol. 14:264-270 (1974).

3. J. G. Wagner. Drug accumulation. J. Clin. Pharmacol. 7:84-88 (1967).

4. J. C. K. Loo and S. Riegelman. New method for calculating the intrinsic absorption rate of drugs. J. Pharm. Sci. 57:918-928 (1968).

5. J. G. Wagner. Method of estimating relative absorption of a drug in a series of clinical studies in which blood levels are measured after single and/or multiple doses. J. Pharm. Sci. 56:652$653(1967)$.

6. J. G. Wagner, P. G. Welling, and A. J. Sedman. Plasma concentrations of propoxyphene in Man. II. Pharmacokinetics. Internat. J. Clin. Pharmacol. 5: 381-388 (1972).

7. L. Z. Benet. General treatment of linear mammillary models with elimination from any compartment as used in pharmacokinetics. J. Pharm. Sci. 61:8-10 (1972).

8. D. Perrier and M. Gibaldi. Relationship between plasma or serum concentration and amount of drug in the body at steady state upon multiple dosing. J. Pharmacokin. Biopharm. 1:17-22 (1973). 